\title{
Big data from social media and scientific literature databases reveals relationships among risk management, project management and project success ${ }^{1,2}$
}

\author{
Maria Papadaki ${ }^{2}$, Nikolaos Bakas ${ }^{4}$ Edward Ochieng, ${ }^{2}$ Ioannis Karamitsos ${ }^{3}$ and Richard \\ Kirkham ${ }^{1}$ \\ ${ }^{1}$ Univerity of Manchester, UK. ${ }^{2}$ British University in Dubai, UAE. ${ }^{3}$ Rochester Institute \\ Technology, Dubai, UAE. ${ }^{4}$ School of Architecture, Engineering, Land and Environmental \\ Sciences, Neapolis University Pafos, Paphos, Cyprus;
}

Corresponding author: Maria Papadaki (maria.papadaki@buid.ac.ae)

\begin{abstract}
The literature review highlights that previous studies have been identifying risk management as an essential tool for project management and could increase the chance of successfully meeting project objectives. In addition, as found from the reviewed literature, risk management has been seen as a tool of allowing the project team to communicate risk information, so as to enhance the decision-making process towards balancing threats and opportunities. Thus, this research aims to examine participants' views on the alignment of risk management, project management and organizational project success. Machine learning algorithms are employed to explore collective data from posts on twitter in order to obtain valuable knowledge about discussions regarding risk management, and project management. Additionally, the corresponding scientific literature obtained from Scopus database was analyzed utilizing bibliometric tools, in order to investigate diverse perceptions in academia and industry. Findings of this study will have implications for practitioners' perception of project risk management.
\end{abstract}

Keywords: Risk management, Project success, Decision making, Project management, Machine Learning, Big Data, Bibliometrics.

\footnotetext{
${ }^{1}$ Second Editions are previously published papers that have continued relevance in today's project management world, or which were originally published in conference proceedings or in a language other than English. Original publication acknowledged; authors retain copyright. This paper was originally presented at the 6 th Annual University of Maryland PM Symposium in May 2019. It is republished here with permission of the author and conference organizers.

${ }^{2}$ How to cite this paper: Papadaki, M., Bakas, N., Ochieng, E., Karamitsos, I., Kirkham, R. (2019). Big data from social media and scientific literature databases reveals relationships among risk management, project management and project success; presented at the $6^{\text {th }}$ Annual University of Maryland Project Management Symposium, College Park, Maryland, USA in May 2019; PM World Journal, Vol. VIII, Issue VIII, September.
} 


\section{Introduction}

In this work, we investigated significant concepts surrounding the project management (PM) and risk management (RM), the related concepts, as well as whether any relationship among them exist in academia or professional practice. Increasing the understanding of the relation between project management, risk management and people perception will lead us to gain knowledge about the use of the risk data regarding the decision making process. This will contribute on building project risk aware culture. Shi in [1] argues that proper implementation of project management, creates added value holistically to an organisation, both in strategic and operation level. In other words, effective risk management must be defined broadly in order to avoid strategic failures, that may lead operational ones. In addition, Drew et al [2] introduce five integrated elements that underpin a firm's ability to manage risks, engage in effective corporate governance, and implement new regulatory changes: Culture, Leadership, Alignment, Systems, and Structure. Benjamin Franklin in 1748 when offering advice to a young tradesman; said "Remember that time is money". The definition of this statement is very pragmatic; any delay to project execution by unexpected or unpredictable factors makes cost increase, thereby directly affecting one another.

Risk is a subjective concept, highly related to people's perception, and many times extremely rare phenomenon occur, which cannot be quantified by statistics or forecasting methods [3]. This may be an outcome of tradition, attitude, and perception. People have a different behavior to the way that they perform risk management; some of them do it more than others or others do not do it at all, because is seen as unnecessary overhead [4]. Hence, human perception in the execution of the risk management process plays a vital role in the successful deployment of the process.

It is essential that decisions taken in a project take account of the level of risk in the project. Therefore, risk management plays a vital role throughout decision making process. Project managers and all the parties that are affected by risks have to be fully aware of the risk and their impacts on the project objectives. A primary characteristic of a decision according to Charette [5] is that "a decision process must be visible, repeatable, and measurable". In the decision process, project members have to make decisions with consideration of risk in a daily basis. Therefore, Risk management and more generally risks have to be a part of daily thinking and know-how. It is like a culture, project members have to be fully aware of risk and the consequences positive or negative and be able to manage or mitigate them in the most efficient way.

(c) 2019 Maria Papadaki, Nikolaos Bakas, Edward Ochieng, loannis Karamitsos, Richard Kirkham www.pmworldlibrary.net

Page 2 of 18 
The willingness of contracting parties to bear risks depends on the factors listed below [6]:

- General attitude to risk; each parties' preferences for different risk/returns trade-offs

- Perception of project risks

- Ability to confront the consequences of a risk occurring

- Ability to manage the associated uncertainty and thereby mitigate the risk

- Need to obtain work: will affect willingness to take risk directly

- Perception of the risk/return trade-offs of transferring the risk to another party.

In particular, Barber [7] explains the influences and interrelationships affecting responsiveness of risk. The following Figure 1 shows factors such risk information, individuals understanding, accountabilities, risk rewards that influence decision making process and therefore increase the likelihood of project success, therefore Barber's work is indicating that there is a direct relation between the concepts of risk, decision making and project success.

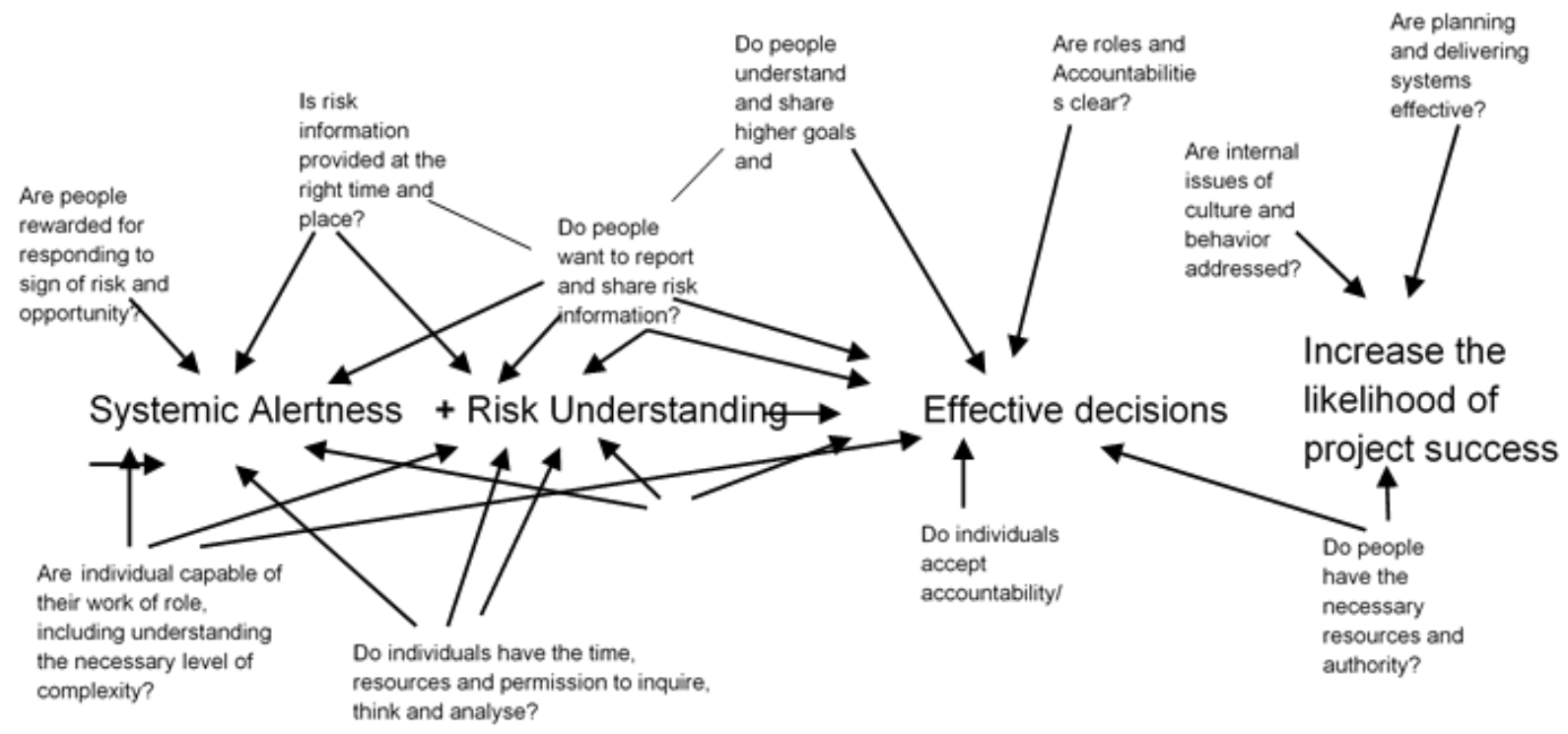

Figure 1: Responsiveness split into understanding of effective decision making

In general, the perception of success is changing nowadays from the traditional iron triangle that focused on time, cost and quality. Recent examples are the mega-project of Burj Khalifa where the plan cost was \$M876 and the final cost reached \$B1.5. Although the above examples of projects do not satisfy the iron triangle and some of the stakeholders' expectations, they are still considered as efficient finally. Therefore, how project success perceived is more a philosophical 
question that can be analysed from different aspects. According to Turner and Zolin [8], a project should be evaluated beyond its immediate completion, while mega projects will have a wider range of stakeholders making judgments. This work investigates the relation between project success and risk management, which go beyond the traditional iron-triangle, to the existence of the project. Risk management is an essential tool through the traditional project life-cycle, but also beyond its initial completion. Therefore, perceptions of people influence decision making process at the modern life cycle of a project, highly affecting its success. To this end, in this work, a big data investigation was performed, utilizing Scopus [9] and twitter [10] databases as well as machine learning algorithms for their analysis.

\section{Bibliometric Literature Review}

\subsection{Machine Learning Deployment for Project Success with focus on Project Management}

In order to investigate a large database of papers on project success, and reach reliable conclusions respectively, this work deployed the use of machine learning algorithms. In particular, a novel bibliometric procedure developed in [11] was utilized to analyze the current work's database. This will demonstrate the terminologies that used alongside with project success, as well as their inter-associations. Therefore, a database was developed from Scopus for the purpose of understanding and analyzing project success from the literature. As a general concept, project success has received a considerable attention within the project management literature over the last three decades [12].

The database was derived from the Scopus website [9]. The term project success is used as a research term in the field of keyword. This investigation will facilitate the better understanding of the literature on the field of project success. It is important to identify other keywords and make a final database closer to the project success. As a first step a database with 2822 papers was identified. For this analysis the database was comprehensively checked and limited to publications with project management focus, therefore the query was limited by choosing specific keywords from the drop down menu which was more relevant for this investigation. Final results constituted a list of 1.243 items from journals, books and peering review conference proceedings. The database can therefore be considered as a reliable material for further analysis with text mining machine learning and tools. Next step was to extract the basic keywords included in the database.

Due to the vast contained information, regarding the keywords, the authors decided to make a further classification of the keywords using fundamental of the project management theories and available approached from the literature. Projects where classified using Newton [13] approach.

(C) 2019 Maria Papadaki, Nikolaos Bakas, Edward Ochieng, loannis Karamitsos, Richard Kirkham www.pmworldlibrary.net

Page 4 of 18 
According to Newton there are two categories the engineering and management projects. Where the first encompass civil, electrical, mechanical, where the deliverables are physical objects such as a bridge or engine. The second category refers to thing like developing an IT system where the final result is not considered to be physical item but a process or a system. DeWit [14] top success criteria were also used as generic classification (Delivering on time, cost and budget). On main limitation of the database was that there are not standardised project management keywords so this is make it very difficult and time consuming for researchers to make relations of terminologies.

The authors found it useful to also include the standard terminology from the PMI and APM associations. Accordingly, the 10 PMI knowledge areas will be used as a further categorization among with the behavioral and the contextual competencies adopted by APM (2008). In addition, because this research is based on actors the role of project manager was also considered as separate category for the classification.

\subsection{Numerical Procedure}

The next Table 1 demonstrates the computational process for the constitution of the bibliometric map. The procedure initially reads programmatically the author keywords column from the *.csv file -exported from Scopus- and calculates of the co-occurrence table of the keywords (Figure 2). Through the constitution of the co-occurrence table the simultaneous existence of the keywords is demonstrated. The colors of the map indicate the amount of co-existence of the keywords. For example, in Figure 2, it is depicted that project management and project success, exhibit major co-occurrence (yellow color), the project risk management with technologies and it processes immense, project performance and project stakeholder management with project success high, project manager and project team with project success medium, and, governance of project manager/ top management support, communication, complexity and strategy with project success as well as with almost all the other existing keywords, low. 


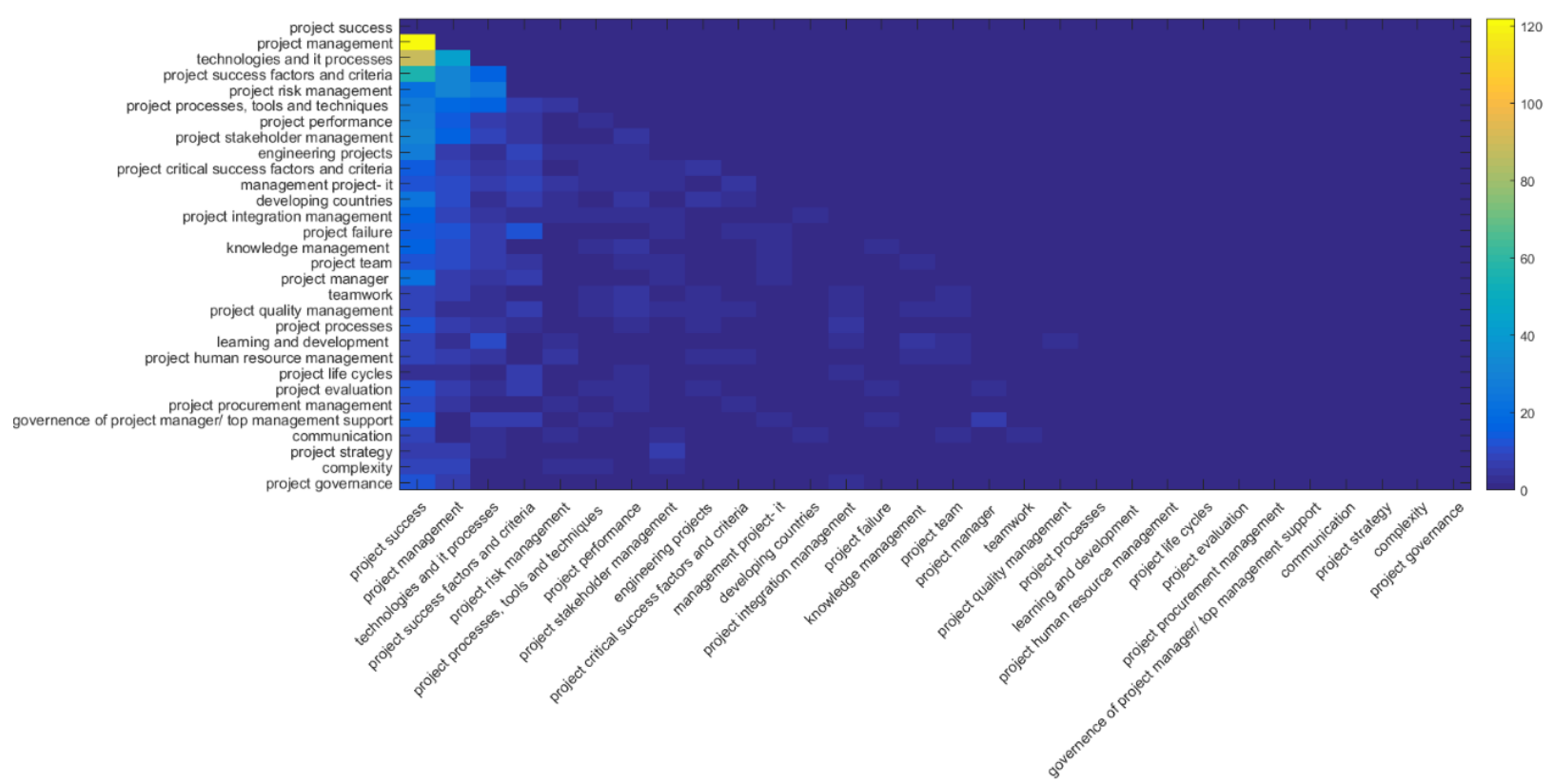

Figure 2: co-occurrence colored matrix

Consequently, the similarity matrix is formulated, and the dis-similarity matrix is computed by inversing each component of the similarity matrix. Accordingly, the optimization algorithm, initialize randomly the positions $\mathbf{p}=\left(x_{i}, y_{i}\right)$ of each item (keyword), and calculates the distances among the features on the bibliometric map. The objective function is the norm of the deviation between the pairwise distances, and the matching dis-similarities. After a vast amount of optimization steps and objective function calculations, the optimal values of the positions xi are exploited to depict the outcomes on the bibliometric map.

Table 1: Algorithm for the Clustering

1. $\mathrm{c}_{\mathrm{ij}}:=$ contingency table (co-occurrence of objects)

2. $\mathrm{s}_{\mathrm{ij}}:=$ similarity

3. $\mathrm{ds}_{\mathrm{ij}}:=\frac{1}{\mathrm{~s}_{\mathrm{ij}}}$ (dis-similarity)

4. $d_{i j}=\left\|x_{i}-x_{j}\right\|$ (distance on map)

5. $\mathrm{f}_{\mathrm{ij}}:=\left|\mathrm{ds}_{\mathrm{ij}}-\mathrm{d}_{\mathrm{ij}}\right|$ (objective function)

Optimization Algorithm

6. Optimality criteria satisfied?

NO YES

7. End $=>$ drawing of bibliometric map 
The bibliometric map represents each item (keyword), located in a point on the 2-Dimensional drawing, with $(x, y)$ coordinates. The entities (keywords), which found to co-occur, are linked though a line, with width proportional to the co-occurences, that is to say the similarity (link strength) between the objects. The distance among the items indicate their dis-similarity, which is written in the middle of each link (exact value), with a suggestive arrow (->), while the corresponding dis-similarity is reported in parenthesis. Finally, each object's font size is equivalent to its number of occurrences, in order to direct identify the most important objects (keywords).

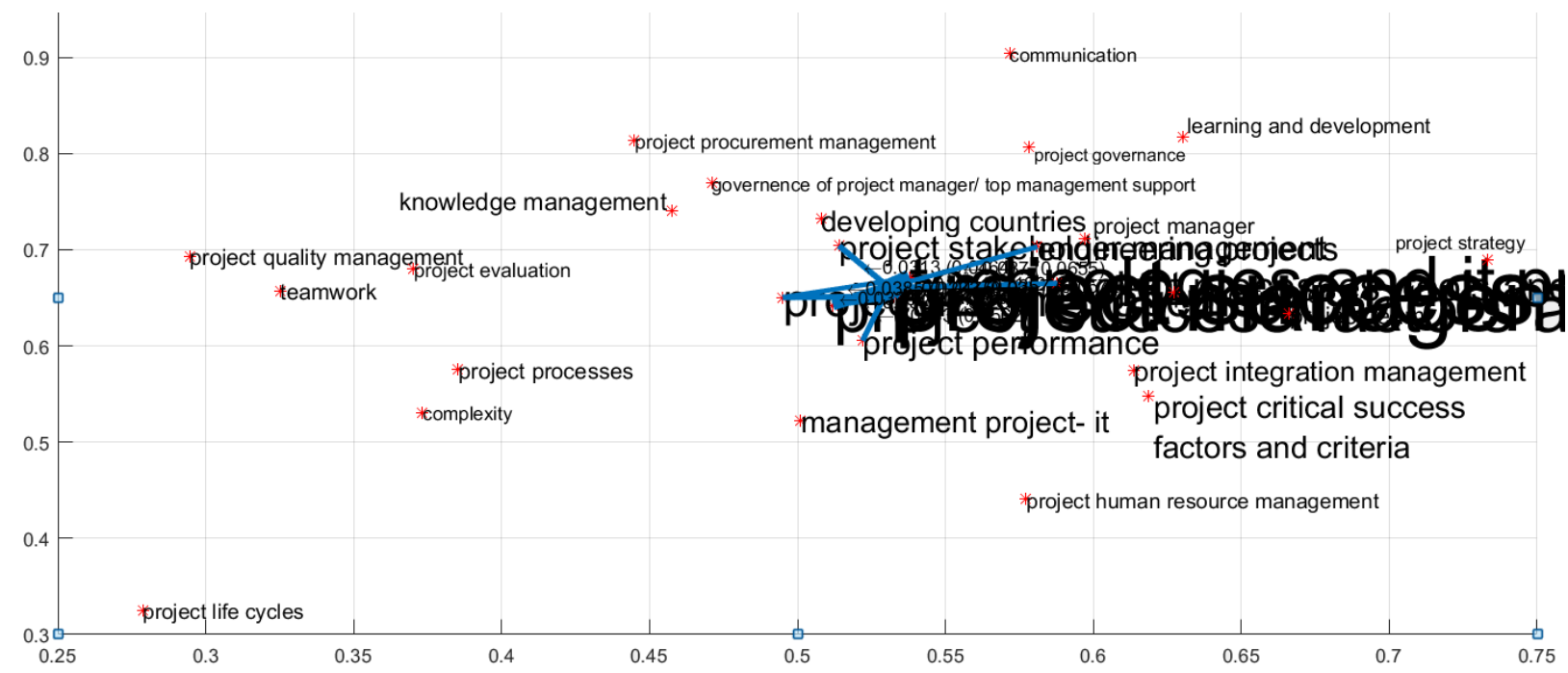

Figure 3: Overview of project success keywords bibliometric map

Consistently with the above described procedure, in Figure 3, the overview of the database regarding project success keywords, bibliometric map for the thirty top keywords existing is demonstrated. The map is optimally consistent with the co-occurrence matrix as described previously. However, it contains significant information depicting the overall associations of the keywords studied, as the relative positions of the keywords on the bibliometric map, is an indicator of the conceptual association among them. For example, in Figure 4, in the center of the map, the keywords project success, project management and technologies and IT processes were identified. Furthermore, the keyword project success is highly associated with keyword engineering projects, which was previously described by Newton [13], classification theory approach, were deliverables are physical objects. Another important relationship that was indicated on the bibliometric map, is that a significant amount of research papers was mentioning developing countries. Furthermore, the keywords project manager and project stakeholders are highly related to the project success as well, because they were positioned in the central region of the map. 


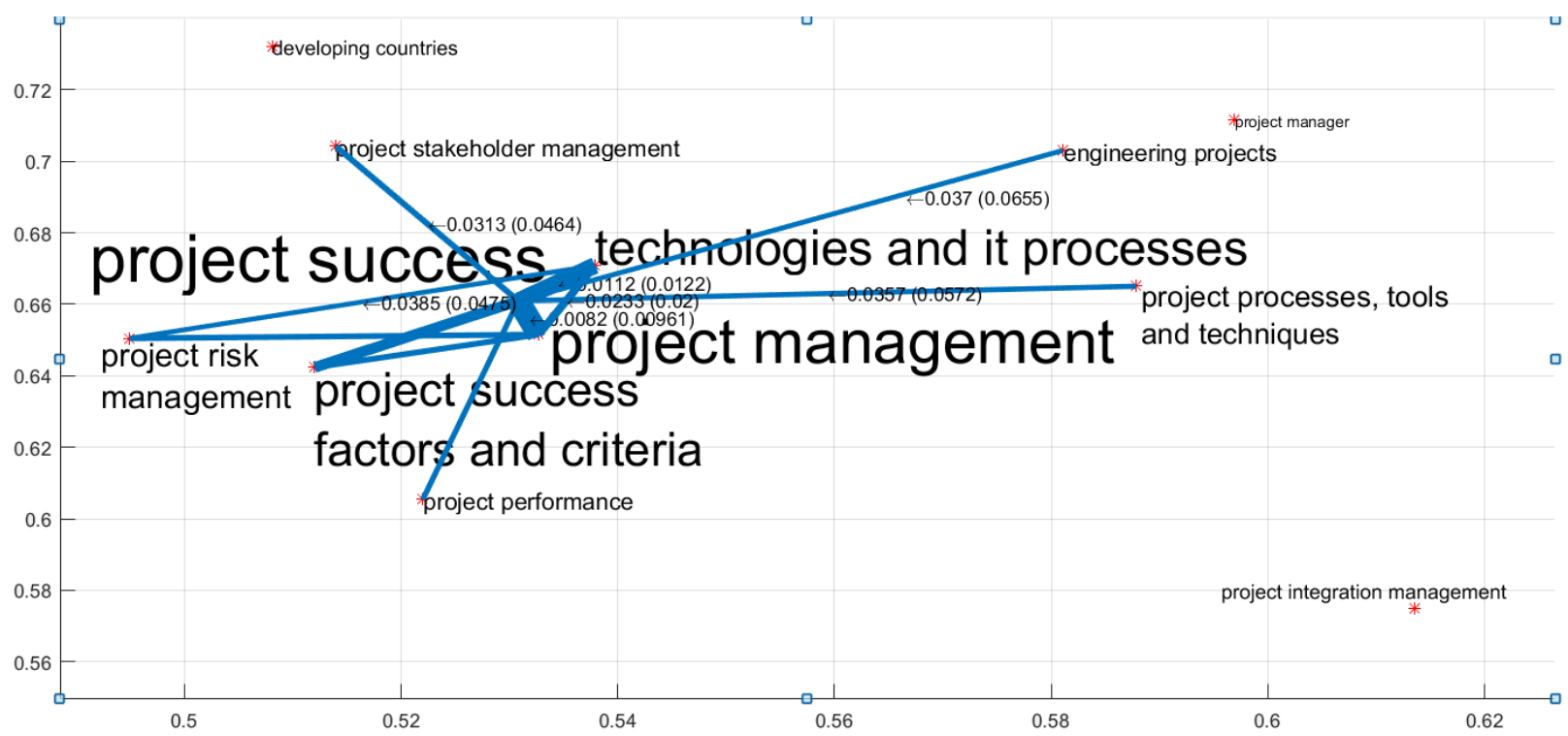

Figure 4: Focus of bibliometric map top keywords

Finally, Figure 4, which is a focus of the core area of Figure 3, reveals that the keyword project risk management, is highly associated with the keyword project management. Because this analysis is based on a rigorous computational methodology and on adequate database of peer reviewed papers it can be reliably to conclude that in scientific literature on project success the concept of project management is studied simulated with the concept of risks management. This finding is contradictory from the later analysis from twitter. Therefore it is proposed to practionares to investigate more on the importance of risk management in the project context. While literature demonstrates the importance, people discussion from social medial does not perceive risk management as an important discussion when referring to projects.

\section{Text Mining using twitter data}

Text mining is an application of data mining to find patterns in the text [15]. In addition, the text mining provides a valuable business decisions insight, from text-based content such as word documents, emails and postings on social media streams as Twitter, Facebook and LinkedIn. For data analysis, public user-generated content derived from Twitter, a text sharing social media network, is used [16]. In our case, we have collected tweets regarding the project management, risk and risk management as well as the relationship among these keywords. The data analysis process consists of a) data acquisition, b) data cleansing and storage, c) data querying and filtering and d) data visualization. For the data acquisition process, the system communicates with Twitter repeatedly through their RESTfulWeb Services (RESTful APIs) to collect all the 
new generated data in an unstructured form, such as post text and language, image URLs, likes, shares, dates and times, etc.

After the acquisition, data cleansing and transformation is needed to select the required data and transform them into a structured form to be stored in the SQL database. The data from the main SQL database table (holding all the collected and transformed records) is also transferred to a cloud data warehouse. Data visualizations can reveal popularity and sentiment for specific hashtags during the examining period. Using text mining/sentiment analysis it is found that positive sentiment dominates during that period, while the negative posts are at low levels [17]. Data was collected approximately for two months (start 24/01/19- finish 26/03/19). A total of 99.894 posts tweets from 32.775 users, 46797 likes and 671.938 shares (Figure 5).

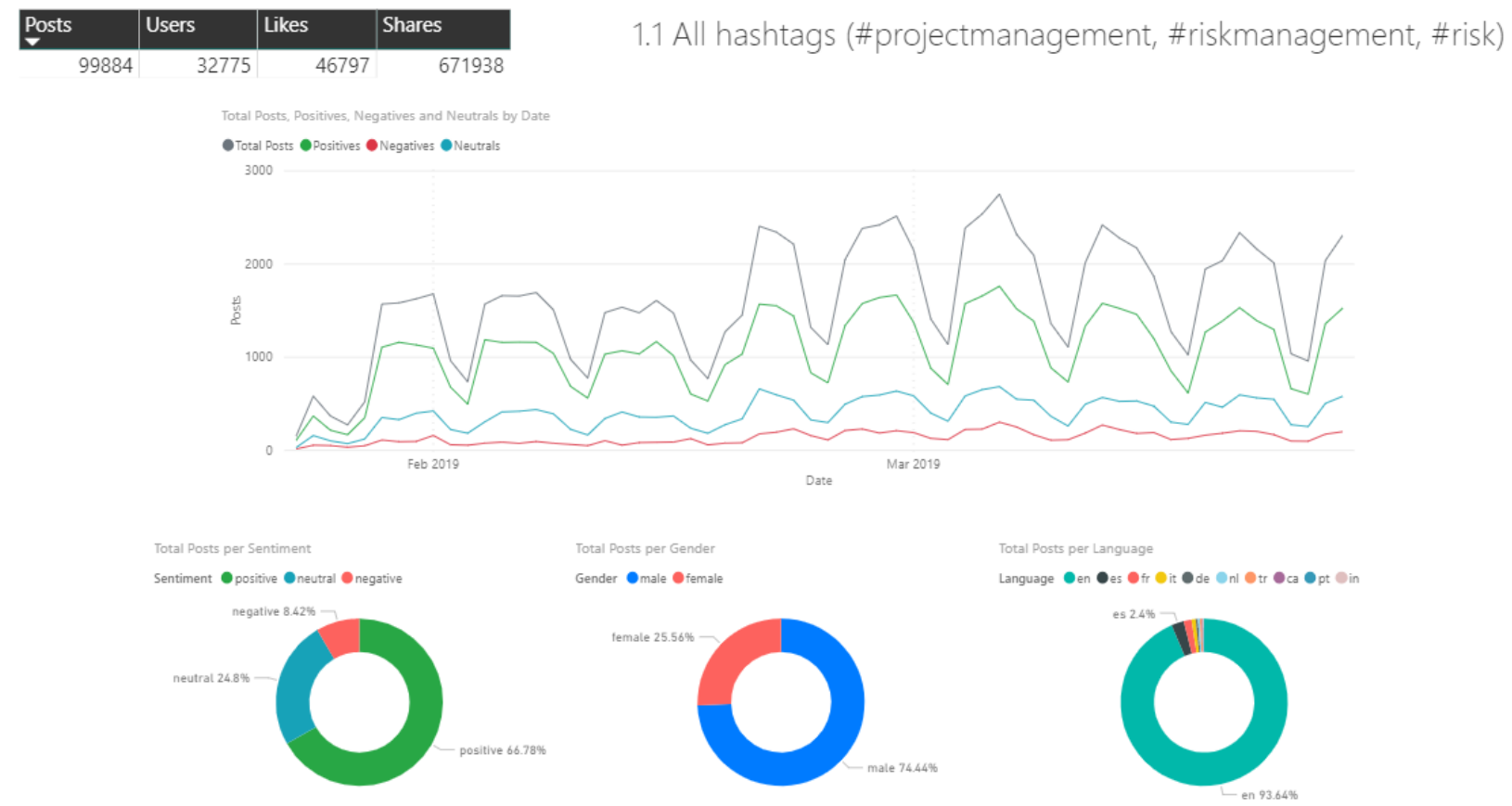

Figure 5: All hashtags - Overview

Figure 5 demonstrates a generic overview of all the results gathered from the search of project management (risk/risk management). It is important to note here that the authors tried to investigate on using the project success keyword; however, the obtained results were insufficient for big data analysis. Therefore, the research concentrated only on the keywords mentioned above. 
PM World Journal

Vol. VIII, Issue VIII - September 2019

www.pmworldjournal.net

Second Edition
Big data from social media and scientific literature databases reveals relationships by M. Papadaki, N. Bakas, E. Ochieng, I. Karamitsos, R. Kirkham

In particular Fig.4 presents the proportion of positives negative and neutral words appeared in the study tweets. It is worth mentioning that utilizing sentiment analysis, the majority of the studied tweets contained positive words (66,78\%). Additionally, the posted tweets were obtained mainly from male users $(74,4 \%)$ rather than female $(25,56 \%)$ indicating that males are more active project management practitioners on sharing their opinion through social media. Finally, it was revealed that the tweets were consisted by different languages such as English, Spanish, French, Italian and German words. However, the majority of tweets are posted in English (93,64\%)

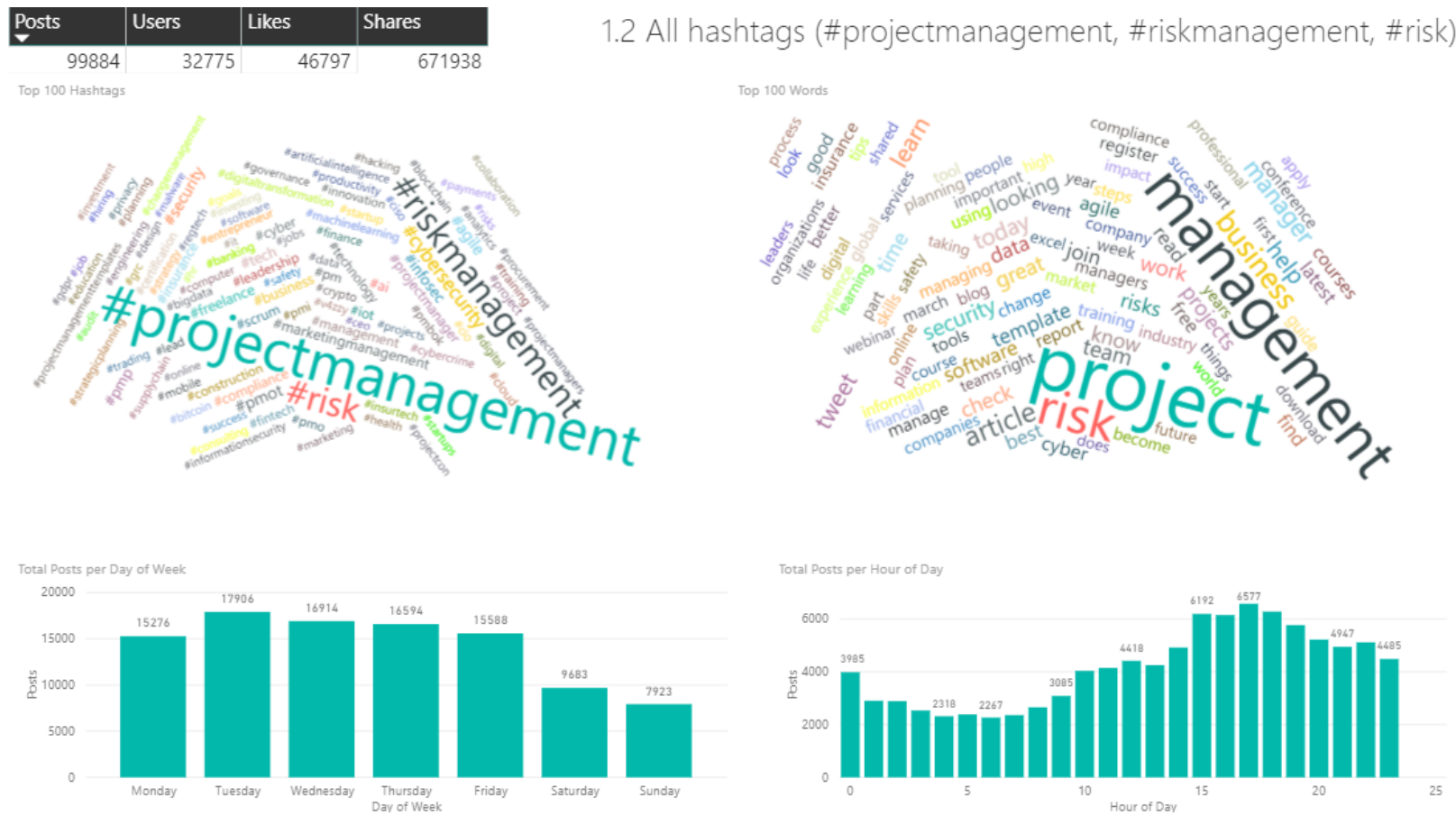

Figure 6:All hashtags - word clouds

A further analyses of the days of the week and the peak hours that posted the tweets was also investigated. The results are presented in Figure 6, highlighting that the tweets were posted on working days as well as working hours. Limited activity has been shown on weekends. Figure 6 also depicts the word clouds of the hashtags and the words used to formulate the message. 


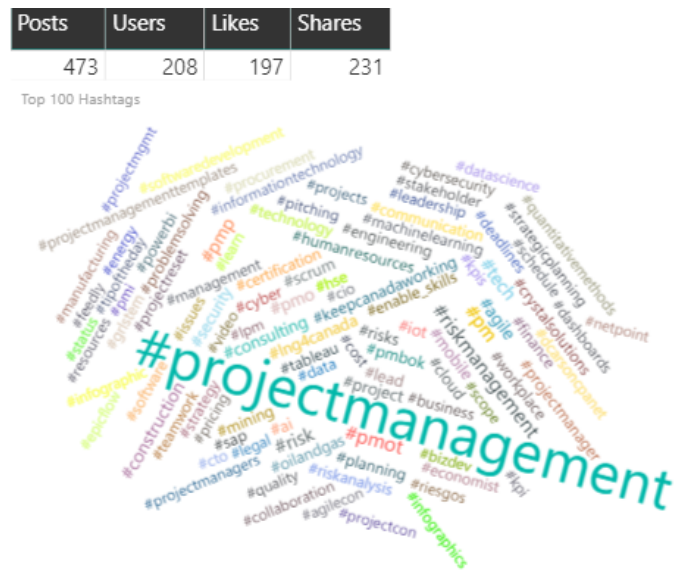

4.2 \#projectmanagement AND (risk OR riskmanagement)
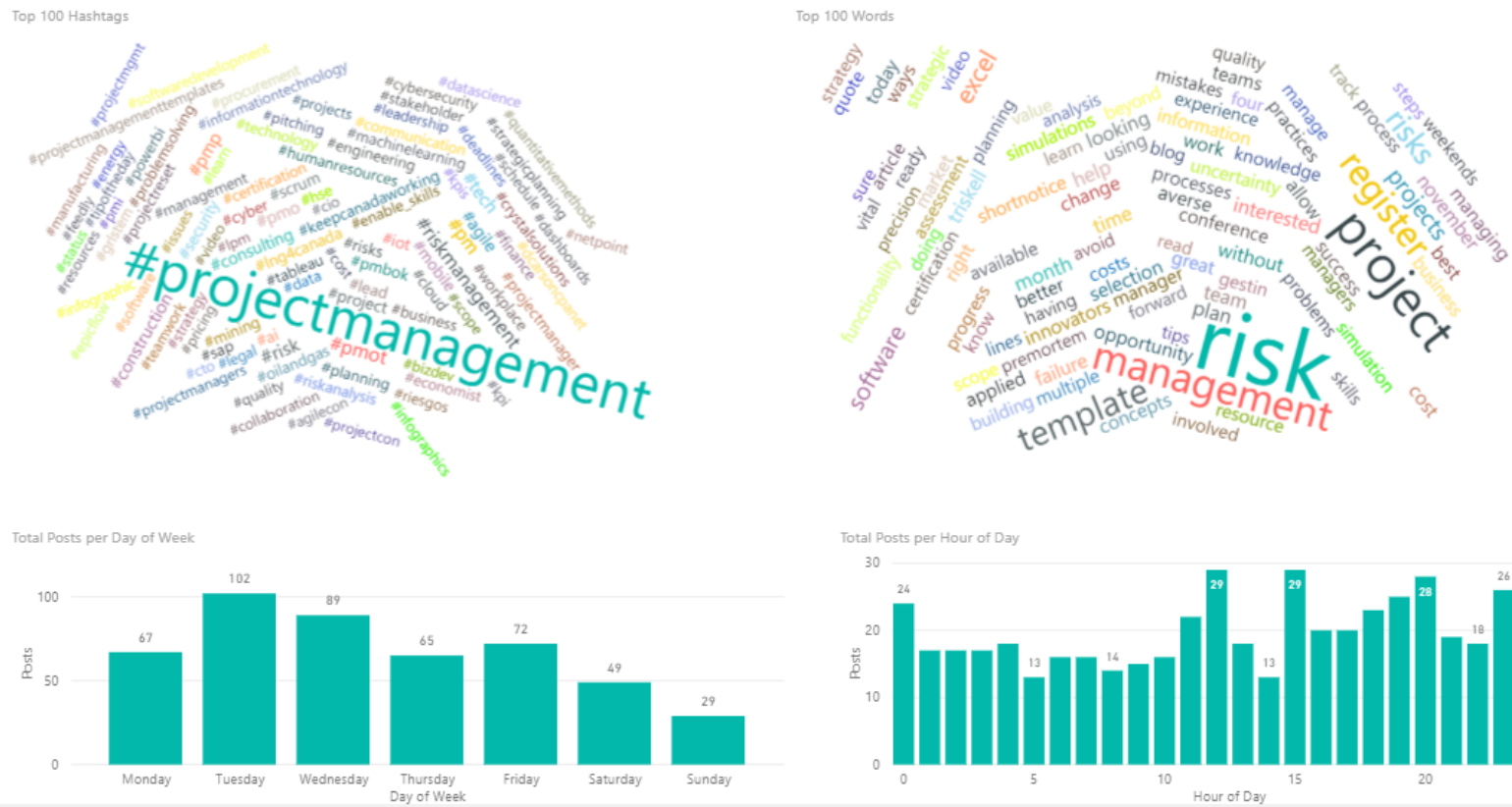

Figure 7: Query for \#projectmanagement AND (risk OR riskmanagement)

Further study demonstrated a deeper knowledge about the content of the tweets (Figure 7). In particular, when the hashtags \#projectmanagement AND (risk OR riskmanagement) where combined in the database query then the resulting posts where 473 only, published by 208 users with 197 likes and 231 shares. It is revealed that on the common word cloud the most popular hashtags are \#Cyber, \#data, \#machinelearning, \#engineering, \#riskanalysis, etc. In addition, in the same Figure 7, some of the most popular words are cost, software, innovation, register, change, resource and knowledge. 

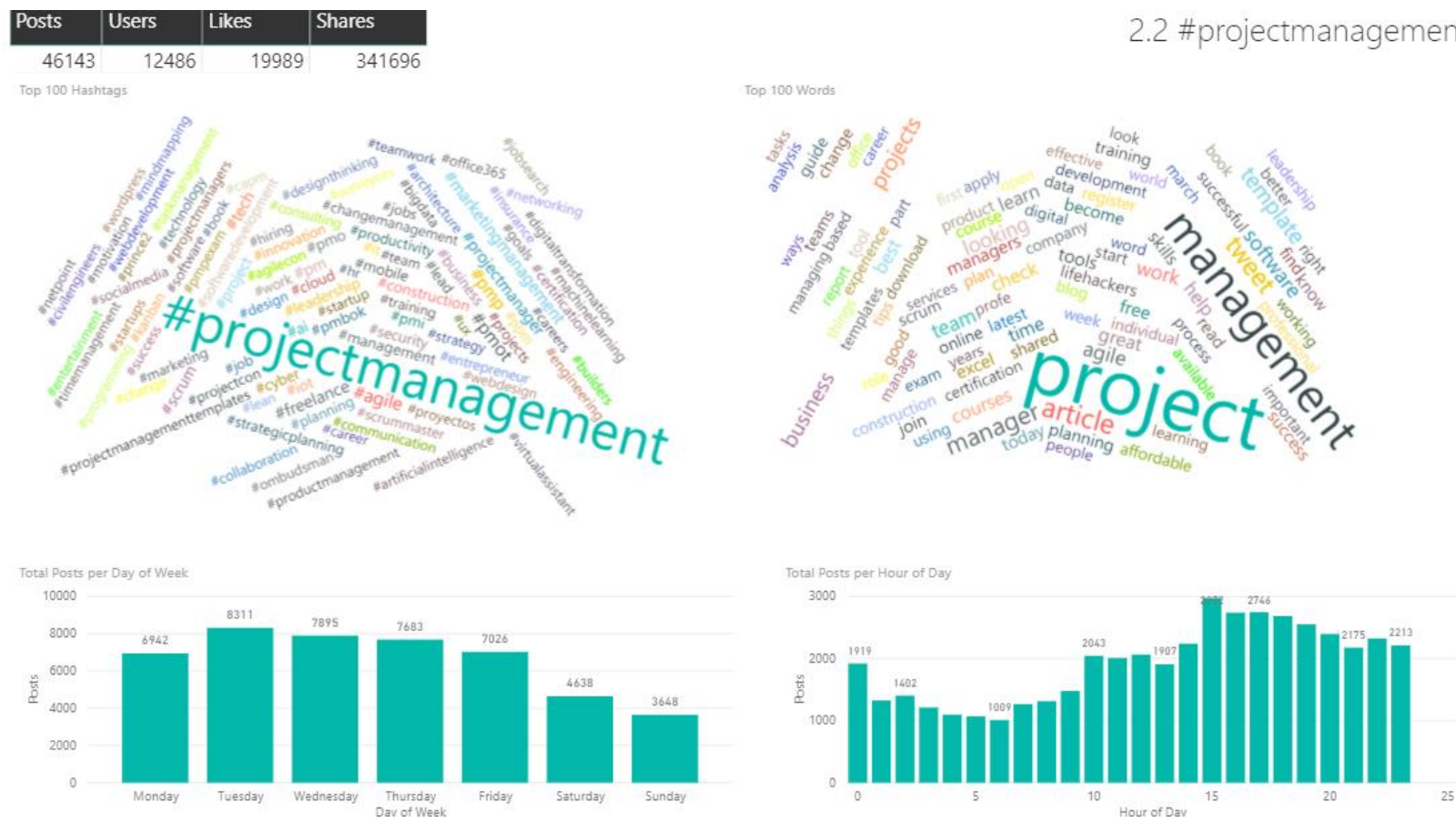

Figure 8: Query for \#projectmanagement

Finally, it is important to show data revealed separately from the area of project management. Figure 8, shows that some common hashtags were \#artifitialintelligence \#planning, \#leadership, \#construction, \#technology, \#engeneering, \#mashinelearning, \#security, \#productivity, etc. Through this analysis it was revealed that risk or risk management was not in the most popular hashtags when people are discussing project management. This is contradictory with the previous research done in Scopus, where academic research in project management is very closely related to risk management. Therefore, it could be argued that practitioners' perception about the importance of risk into project are limited. The question is why practitioners are not engaging with the importance of risk in the project context; when most of them think that it is important. Do really practitioners do proper risk management; and if they do actually why its benefits is an open question. The challenges are to investigate why if so important is not discussed enough. This work shows that there is a gap between academic and public perspective in the importance of the use of risk management.

Through the above analysis, we could argue that engineering, and IT have a significant influence in project management, both in academic publications and people's discussions in twitter. The fourth industrial revolution exhibits a high impact in projects, as revealed by the corresponding discussions. Similarly, risk, is highly associated with the same concepts, of cyber-security, artificial intelligence, big data analytics and relevant topics. 


\section{Conclusions}

Risk management and more generally risks have to be a part of daily thinking and know-how. It is like a culture; project members have to be fully aware of risk and the consequences positive or negative and be able to manage or mitigate them in the most efficient way. This paper was an investigation in the area of project management and risk management insights from academic and practitioner's risk management in relation to project success. As a general concept project success has received a considerable attention with the project management literature over the last three decades [12]. This research utilized data from social media (twitter) and from an academic literature database (Scopus). Hence, in order to investigate a large database of academic papers on project success, and reach reliable conclusions, this work deployed in the research methodology the use of machine learning algorithms. In particular, a novel bibliometric procedure developed in [11], was utilized to analyze the current work's database. Then in order to identify different patterns and practitioners' opinions, the research looked on data derived from twitter and analysed it using big data analytics techniques. The present study provides additional evidence with respect to the relation between risk management, project success and people's perception.

In particular, from the study on Scopus, it was found that project management, project success and risk management are highly associated in academic research. In addition, the same data, revealed that publications are more generated from the engineering and it fields, as the top keywords appearing in the Bibliometric map which is highly related to project success, project stakeholders, the project manager, process tools and techniques. Furthermore, it is encouraging, that projects from developing countries, highly contribute to the academic research. The second analysis, with twitter data, verify Engineering and IT sectors' importance in formulating public opinions. Also, discussion on project management, mainly concern topics as tools, planning, learning, skills, big data and people. The deeper investigation regarding the relation among project management and risk or risk management, as attained by a specific database query, shown that 99884 total tweets, were limited to 473 tweets only. This further underlines the insufficient use of risk data in people's decision making with respect to project management. This work is a part of an ongoing research on increasing the awareness of people towards a risk aware culture. 


\section{References}

[1] Q. Shi, "Rethinking the implementation of project management: A Value Adding Path Map approach,” Int. J. Proj. Manag., 2011.

[2] S. A. Drew, P. C. Kelley, and T. Kendrick, "CLASS: Five elements of corporate governance to manage strategic risk," Bus. Horiz., 2006.

[3] S. Makridakis and N. Bakas, "Forecasting and uncertainty: A survey," Risk Decis. Anal., vol. 6, no. 1, pp. 37-64, Jan. 2016 [Online]. Available:

https://content.iospress.com/journals/risk-and-decision-analysis/6/1

[4] M. Papadaki, A. W. Gale, J. R. Rimmer, R. J. Kirkham, A. Taylor, and M. Brown, "Essential Factors that Increase the Effectiveness of Project/Programme Risk Management," Procedia - Soc. Behav. Sci., 2014.

[5] R. N. Charette, "Large-scale project risk management is risk management," IEEE Softw., 1996.

[6] S. C. Ward and C. B. Chapman, "Risk-management perspective on the project lifecycle," Int. J. Proj. Manag., 1995.

[7] R. B. Barber, "Understanding internally generated risks in projects," Int. J. Proj. Manag., 2005.

[8] R. Turner and R. Zolin, "Forecasting success on large projects: Developing reliable scales to predict multiple perspectives by multiple stakeholders over multiple time frames," Project Management Journal. 2012.

[9] "Scopus." [Online]. Available: https://scopus.com/home.uri

[10] "Twitter." [Online]. Available: https://twitter.com/

[11] V. Plevris, N. Bakas, G. Markeset, and J. Bellos, "Literature review of masonry structures under earthquake excitation utilizing machine learning algorithms," in Proceedings of the 6th International Conference on Computational Methods in Structural Dynamics and Earthquake Engineering (COMPDYN 2015), 2017, pp. 2685-2694 [Online]. Available: https://www.eccomasproceedia.org/conferences/thematic-conferences/compdyn$2017 / 5598$

[12] L. McLeod, B. Doolin, and S. G. MacDonell, "A perspective-based understanding of project success," Project Management Journal. 2012.

[13] P. Newton, The Principles of Project Management, Project Skills. 2008.

[14] A. de Wit, "Measurement of project success," Int. J. Proj. Manag., 1988.

[15] S. VijayGaikwad, A. Chaugule, and P. Patil, "Text Mining Methods and Techniques," Int. J. Comput. Appl., 2014.

[16] M. Komorowski, T. Do Huu, and N. Deligiannis, "Twitter data analysis for studying communities of practice in the media industry," Telemat. Informatics, 2018.

[17] K. Vassakis, E. Petrakis, I. Kopanakis, J. Makridis, and G. Mastorakis, "Location-Based Social Network Data for Tourism Destinations," in Big Data and Innovation in Tourism, Travel, and Hospitality, 2019. 


\title{
About the Authors
}

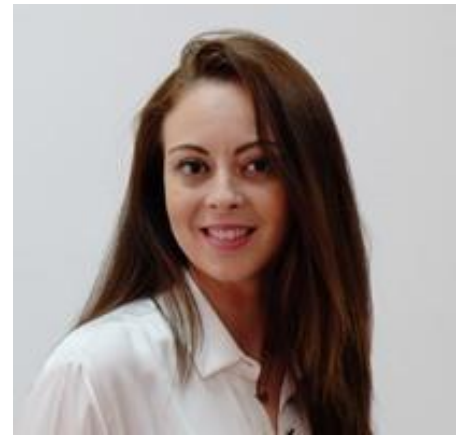

\section{Dr Maria Papadaki}

\author{
United Kingdom
}

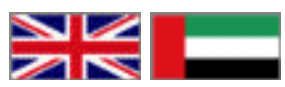

Dr Maria Papadaki obtained her first degree in Business Economics from Salford University. Part of her undergraduate study was carried out in IPAG University in Nice (France). Maria completed her MSc in Management of Projects from The University of Manchester (UoM) in 2005. As an internship, Maria undertook an economic study into the viability of establishing Formula 1 racing in Crete. In 2006, she joined the UoM PhD programme and also worked as a research associate and teaching assistant for the school of Mechanical Aerospace and Civil Engineering (MACE). In 2008, she conducted an internship with the Risk Function in Rolls-Royce plc.. In 2009 was appointed as an Enterprise Risk Management Professional at Rolls-Royce's. In 2011 she joined Rolls-Royce plc. Supply Chain Function as a Risk Manager in order to work cross functionally and across all Rolls-Royce plc sectors (Civil, Defense, Energy, Nuclear and Marine) to design, develop and implement integrated and standardised tools and processes to enable the business to proactively identify, manage and mitigate delivery risks across the global supply chain. In 2013, she completed her doctoral studies in the field of risk from The University of Manchester.

In 2013, Dr Maria Papadaki was seconded as an Interim Head of Risk, leading the risk management for the UK Nuclear Restoration (UKNR), a joint venture between AMEC, Atkins and Rolls-Royce plc bidding for the largest nuclear decommissioning project in UK. In 2014, Maria initiated the idea and led the development of the first innovation Hub (H2B) in Crete, Greece on behalf of the Heraklion Chamber of Commerce. In 2015 Dr Papadaki joined the University of Manchester working as a Senior Relationship Manager for the British University in Dubai and the UK Alliance Universities and also acted as the Head of the Programme Management office and the DCRI (Dubai Centre for Risk and Innovation). She is now working as the Managing Director for the DCRI and an Assistant Professor for BUiD. Under this profile, she has been leading the development of digital certificates for BUiD's graduates which made the university 1st in the Middle East and 3rd in the world implementing Blockchain technology in education. Her research and work are now focused on Innovation, Blockchain, Artificial Intelligence, Enterprise Risk Management and Cyber Security. Finally, in 2018 she was appointed to the Board of Directors for the Institute of Risk Management (irm) in London. Under this profile she is leading the global education and training standards strategy for the institute (irm). Dr Maria Papadaki is a visiting lecturer in The University of Manchester and her research is now focused on innovation, Blockchain, Artificial Intelligence, and Enterprise Risk Management. 


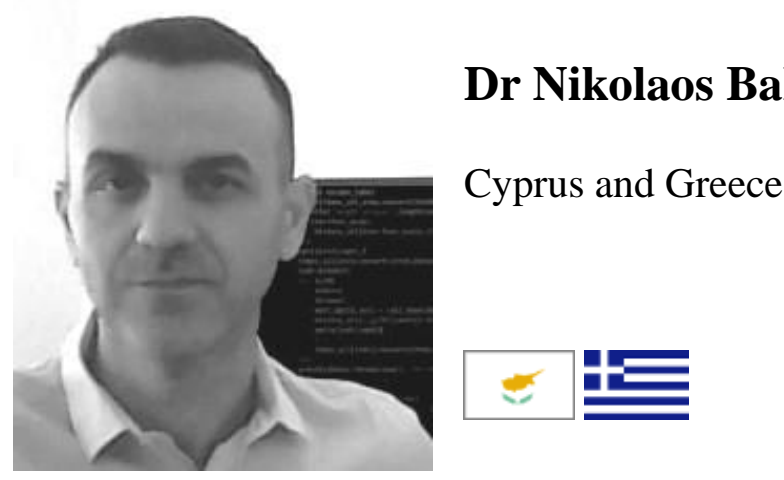

Dr. Nikolaos Bakas is currently a Lecturer in Computational Mechanics at Neapolis University Pafos; External Collaborator of National Technical University of Athens, Director of NOESYS Consulting and External Associate at AXIA Chartered Surveyors. Dr. Bakas' work concerns developing numerical methods and applying machine learning algorithms, in a variety of thematic areas in research and industry, for data analysis, predictive modeling, numerical optimization, quantitative insights, clustering and analysis of literature. In his recent monograph in Research, a Science Partner Journal, he provided a generic numerical solution for the hopelessly ill-conditioned mathematical problem of extrapolation, with prediction horizons $1000 \%$ higher than the existing methods in the scientific literature. He has been teaching for more than seven years in total, in the National Technical University of Athens, Engineering Intelligence and at Neapolis University Pafos, Cyprus, where he is currently appointed as a Lecturer. In the past, he has also founded and managed a lifelong training organization for engineers with more than 5000 trainees. Apart from his teaching and administrative activities, he is focused on research in applied mathematics, machine learning, data science, optimization, and relevant topics. He is also running NOESYS, for seminars, consulting and professional applications of machine learning algorithms.

Career Milestones include A recent monograph in Research, a Science Partner Journal for the numerical solution of the predictions problem of analytic functions; 40 Research works on numerical methods, optimization, data analysis, machine learning, statistics, and predictions; 10 years of professional and scientific programming (MIT's Julia, Matlab, C++, etc.); 7 years of Teaching at Universities, Seminars, and Workshops; Foundation, growth, and management of Engineering Intelligence, an educational organization for engineering seminars with more than 5000, professional Engineers trainees. Continued as noesys.net; and Participation and management of impactful research projects with European funding (i.e. interview at Euronews). He can be contacted at n.bakas@nup.ac.cy, nibas@mail.ntua.gr 


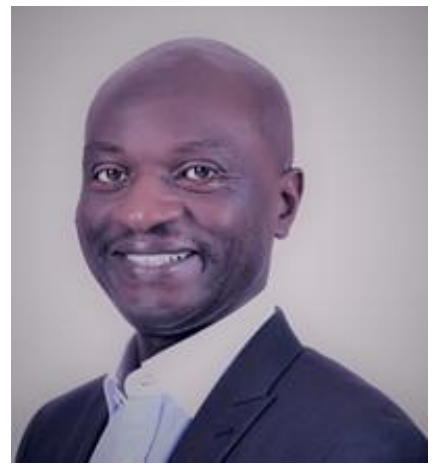

\section{Prof Edward G. Ochieng}

Dubai, UAE

Edward Ochieng, PhD, PGCertHELT, MSc, BSc (Hons), FHEA, AHEA is a Professor of Project Management in the Faculty of Business and Law at The British University in Dubai (BUiD), United Arab Emirates. Dr Ochieng's research is focused on major project management. He has extensive experience and knowledge relating to organisational challenges and solution development for managing large capital and heavy engineering projects. Edward has published 3 books, 28 book chapters and over 85-refereed papers in refereed journals and conferences. He has supervised $9 \mathrm{PhD}$ students to successful completion and over $300 \mathrm{MSc} / \mathrm{MBA}$ industrial projects by research. Edward is currently supervising $4 \mathrm{PhD}$ students. He has also acted as an internal and external examiner of more than $11 \mathrm{PhD}$ students in different UK universities. Edward has secured an estimated $£ 654,538.00$ from a range of funders for several projects and consultancy in project management. Email: Edward.ochieng@buid.ac.ae

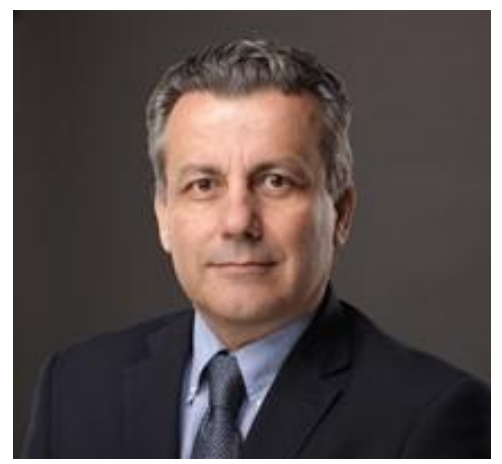

\section{Ioannis Karamitsos}

Dubai, UAE

Dr. Ioannis Karamitsos (B.Sc., M.Sc., Ph.D.) has vast industry and research experience over 25 years as an executive manager who worked within the private and public sectors and experienced within European, Middle East and Chinese companies. From 2010 to present, Ioannis is a Regional Director Technology Consultancy at Orange Business Services. Ioannis is member of DSOA-Dubai Innovation Advisory Board. In 2016, he had joined the Department of Electrical Engineering as an adjunct professor at Rochester Institute of Technology Dubai. In addition, Ioannis is academic coordinator for the Master of Data Analytics provided by RIT Dubai. 
He is particularly interested in applying Blockchain, Cryptography, Machine learning, IIoT, and data mining techniques to emerging problems related to large-scale decentralized cyber-physical systems and critical infrastructures as well as energy, mining, health care and other domains of major economic and social impact. Ioannis received his PhD in Computer Science from University of Sunderland, UK, a Master Degree in Telematics Management from University of Danube Krems, Austria, and Bachelor Degree (Laurea) in Electronic Engineering from University of Rome "La Sapienza", Italy. His have also executive certificates from MIT (Tackling the challenge of Big Data) and from Columbia Business School (Driving Strategic Impact Program).

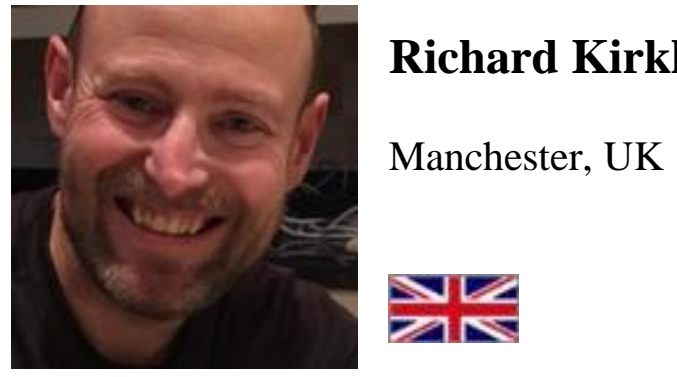

Richard Kirkham is currently co-lead of the Management of Projects Research Group in the School of Mechanical, Aerospace and Civil Engineering at the University of Manchester, with responsibility for research. Richard is an active researcher in the field of project studies; his research and teaching interests focus on risk management in the context of whole-life appraisal of buildings and civil infrastructure. Richard's earlier research contributions in whole-life costing for infrastructure (incorporated into ISO15686-5) continue through government advisory, particularly in the context of major government projects This was recognized in 2016 with the award of an ESRC staff secondment to the Cabinet Office where he worked in the Portfolio Insight Team (now the Analysis and Insight Unit) of the Infrastructure and Projects Authority.

Richard is also an active participant and co-investigator in the ESRC funded 'Project X Improving Project Delivery' (Grant Ref: ES/S009841/1). This is a major investment that seeks to improve the evidence base surrounding major project and programme delivery in government by generate unique insights through world-leading 'co-produced' research. Richard is also an active researcher in the Thomas Ashton Institute for Risk and Regulatory Research, collaboration between The University of Manchester and the Health and Safety Executive.

Richard was PI for two Cabinet Office funded projects examining risk in the context of government transformation. Richard's teaching at Manchester covers a broad spectrum of undergraduate and postgraduate work - including 'Civil Engineering Practice', 'Construction Management Professional Practice' and 'Risk Management'. Richard has also delivered blended learning education through the MSc Project Management Professional Development Programme and the BP Executive Education programme that was led by Manchester Business School. Richard has also held a number of internal appointments including Faculty Employability Lead and Portfolio Director for the British University in Dubai (BUiD). 